\title{
New Considerations in the Design of Microstrip Antennas
}

\author{
Naftali Herscovici, Senior Member, IEEE
}

\begin{abstract}
Printed microstrip antennas and arrays are known to have limitations in terms of bandwidth and efficiency, all imposed by the very presence of the dielectric substrate. Microstrip arrays printed on a very thin film and separated from the ground-plane by foam were successfully designed; however, the fabrication difficulties associated with the use of foam considerably increases the fabrication cost. In this paper, a new concept is presented. Rather than using a superstrata geometry, the "printed circuit" is etched out of metal and supported in "strategic points" by (metallic or nonmetallic) posts. The main motivation for this work was to obtain large microstrip arrays, which exhibit a higher efficiency than conventional ones, and can be fabricated using inexpensive large quantity production techniques. However, this technology was also used to develop many new types of microstrip antennas. Microstrip elements and arrays based on this technology were designed and fabricated at $L, S$, and $C$ bands.
\end{abstract}

Index Terms-Microstrip antennas.

\section{INTRODUCTION}

$\mathbf{T}$ HE dielectric losses associated with the microstrip arrays limits the maximum efficiency that can be achieved by this type of antenna. An excellent study of these losses is given in [1]-[3]. In [3], the losses associated with the feed network radiation and surface waves are also incorporated in the overall efficiency computations. The impact of the dielectric substrate on the printed antenna performance was extensively treated in the literature.

In this paper, a new type of microstrip array is proposed. It consists of a circuit, which includes only the metallization part of the "printed circuit" suspended over the groundplane. Some work was done in the past with small planar suspended microstrip arrays, usually supported by foam [4]. The technique described in this paper deals with a new class of microstrip antennas, which will be shown to open new possibilities in the design approach as well as types of applications. This new approach presents a number of advantages over the classical printed microstrip arrays or any other types of planar suspended microstrip.

1) It permits the use of three-dimensional (3-D) transitions from one level to another so that the width of the lines can be controlled by changing the spacing between the line and the ground-plane; this feature has an impact on the feed network radiation and the coupling between the feed network and the radiating elements.

Manuscript received July 21, 1997; revised December 16, 1997. The author is with Cushcraft Corporation, Manchester, NH 03103 USA. Publisher Item Identifier S 0018-926X(98)04993-X.

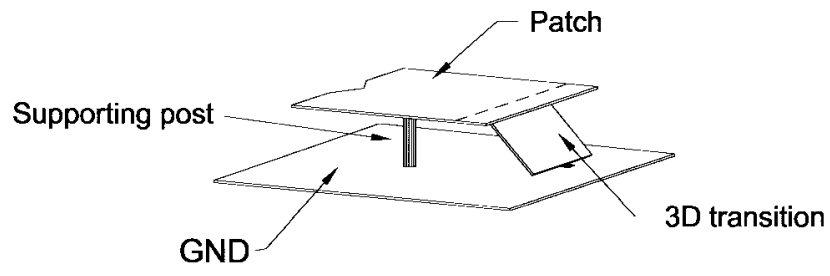

Fig. 1. The suspended patch fed by a 3-D transmission line.

2) The feed network is purely TEM so there is neither dispersion nor surface waves.

3) It supports greater changes in temperature since there is no dielectric (with a temperature expansion coefficient different from that of the metallization).

4) The efficiency is considerably higher, since there are no dielectric losses and no surface wave losses.

5) The main source for blindness in scanning arrays (namely the surface waves) are removed.

6) In some cases, the fabrication cost is considerably lower than that of conventional microstrip since the cost of the dielectric substrate is eliminated.

7) It can handle significantly higher power levels (especially $\mathrm{CW}$ ).

8) It allows for matching techniques similar to those used in waveguide technology.

9) The intermodulation characteristics are improved because of the elimination of the nonlinear effects associated with the dielectric substrate.

\section{The Basic Radiating Element}

The basic radiating element is shown in Fig. 1. It is a microstrip patch cut-out (or etched-out) of sheet metal and supported in the center by a post (metallic or nonmetallic).

It can be fed by another microstrip line, which is either at the same level and parallel to the patch itself [Fig. 2(a)], or oblique [Fig. 2(b)] or even perpendicular to the ground plane [Fig. 2(c)]. In the two latter cases, the feeding mechanism is a 3-D transition. The use of the 3-D transition for the radiating element or the array feeding network presents new degrees of freedom in the design. The most important one is the possibility to control the width of the feeding transmission line. In the classical printed-circuit-type of microstrip antennas, the input impedance of the patch can be very high (or low) involving a very narrow (or wide) feeding line. Here, as shown in Fig. 2(c), the width of the feeding line can change very rapidly and brought to a comfortable size at the appropriate 


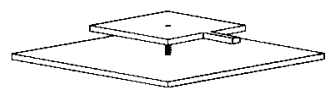

(a)

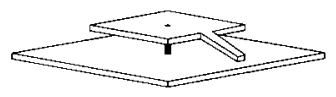

(b)

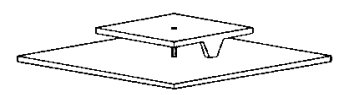

(c)

Fig. 2. The suspended patch fed by a transmission line. (a) Parallel to the ground plane. (b) Oblique. (c) Perpendicular to the ground plane.

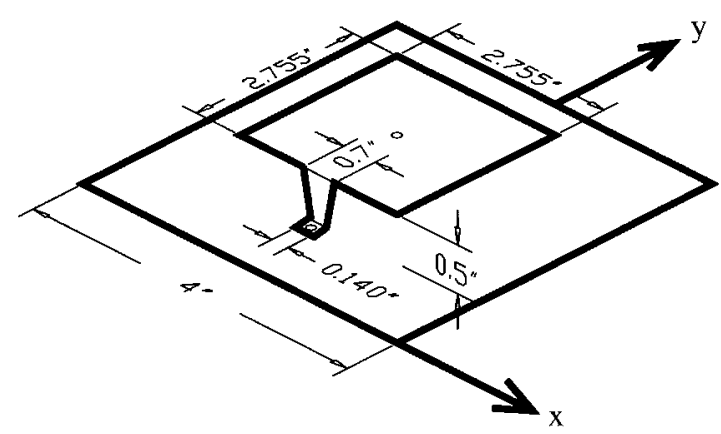

Fig. 3. The dimensions for a patch similar to the one in Fig. 2(c) designed to work in the PCS band $(1.88-1.99 \mathrm{GHz})$.

height above the ground plane. Furthermore, the 3-D transition allows for the design of very efficient patches that are located fairly high over the ground plane (up to a quarter wavelength). This type of patch, known to have considerably high input impedance cannot be fed by a probe (which would be too long and would radiate) or a microstrip transmission line parallel to the patch (which would be too narrow and practically impossible to fabricate). In some applications, the bandwidth obtained with such a patch eliminates the need for a parasitic element.

The patch shown in Fig. 2(c) was built for PCS applications. It has a rectangular shape and is fed by a perpendicular transmission line (see dimensions in Fig. 3). The patch is supported in the center by a plastic post to avoid the need to consider the issues of good electric contact (between the post and the patch/ground-plane) and intermodulation. If the diameter of the post does not exceed about $10 \%$ of the largest dimension of the patch, the impact on the input impedance was found to be negligible even if the post were metallic. This is a well-known fact in the case of a symmetric patch, however, an extensive study was done on the impact of an aperture in the patch on the input impedance of patches of different shapes [6]. In most of the cases studied, involving suspended tall patches, a small aperture in the patch (less than $10 \%$ of the largest dimension of the patch) was found to have a very small or no impact at all on the input impedance, regardless of its location. The measured voltage standing wave ratio (VSWR) of the antenna in Fig. 3 is shown in Fig. 4. The bandwidth (for VSWR $<2: 1$ ) is definitely exceeding the required personal communication system (PCS) band (7.6\%). The patterns measured at $1.92 \mathrm{GHz}$ (Fig. 4) exhibit a relatively low front-to-back ratio due to the size of the ground plane, which was a constraint in this particular design (Fig. 5 shows radiation patterns of the patch described in Fig. 3).

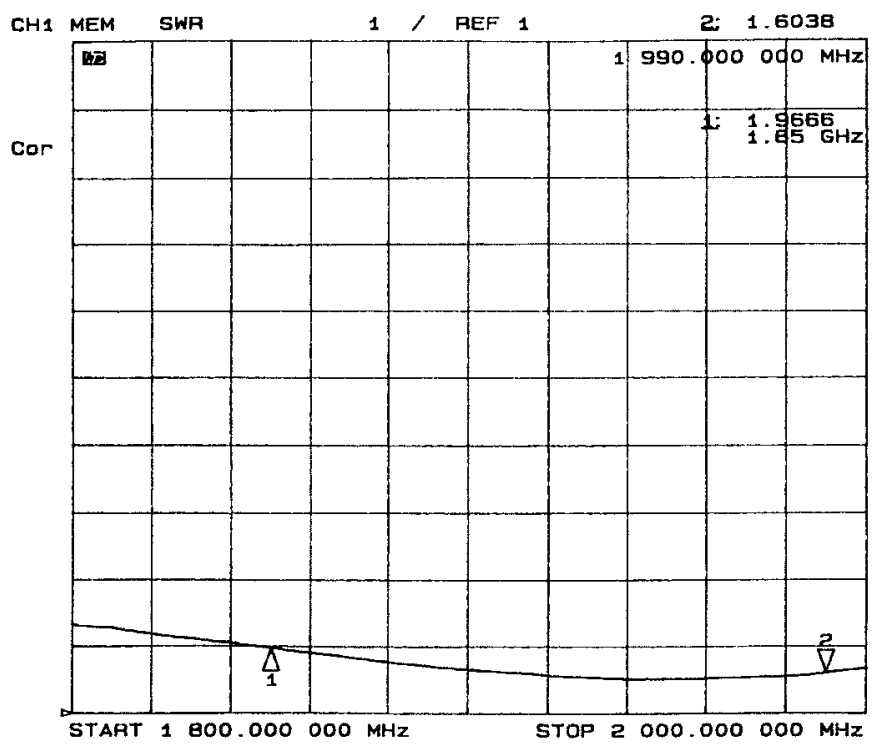

Fig. 4. The VSWR for the patch described in Fig. 3.

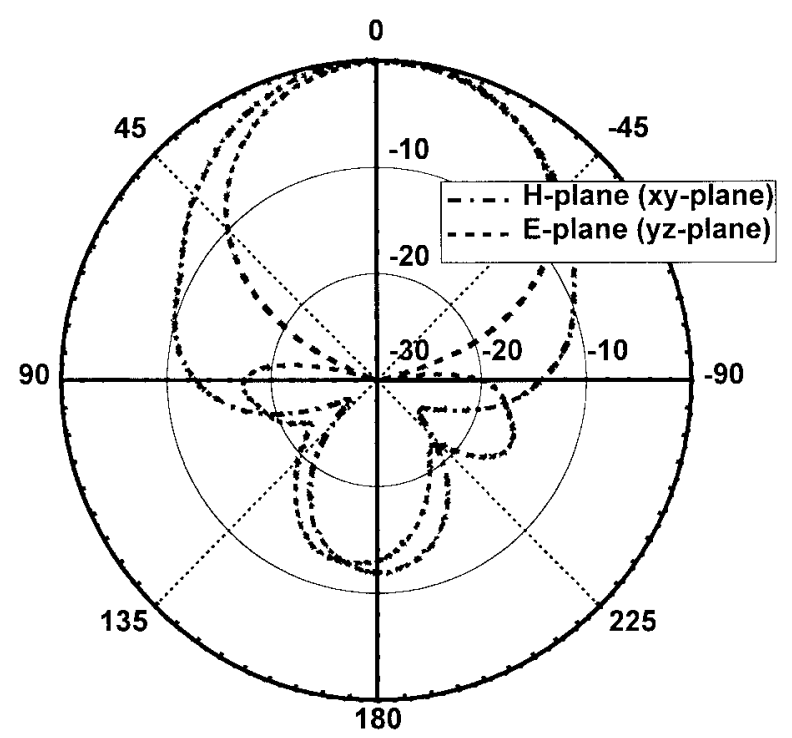

Fig. 5. The radiation patterns of the patch described in Fig. 3.

A circularly polarized version of the previously described patch is shown in Fig. 6. It consists of the patch shown in Fig. 3, fed by two perpendicular feed lines. To save space, the $90^{\circ}$ hybrid is located between the patch and the groundplane. The $90^{\circ}$ hybrid was first designed without considering the presence of the patch. The impact of the hybrid spurious radiation was compensated by a slight modification of the patch size given in Fig. 3. The two probes shown in Fig. 6 feed the input ports of the hybrid; the two output ports of the hybrid are supported by two nonmetallic posts and are connected to the two perpendicular lines feeding the patch. The measured axial ratio of this antenna versus frequency is shown in Fig. 7. The impedance bandwidth (VSWR $<2: 1$ ) is $1.6-2.1 \mathrm{GHz}(>31 \%)$.

\section{FOUR-ELEMENT ARRAYS}

A number of different geometries were generated for a 2 $\times 2$ microstrip subarray, some of which are given in Fig. 8 . 
TABLE I

Design Parameters for the Antennas Shown in Fig. 8

\begin{tabular}{l|l}
\hline \multicolumn{1}{c|}{ Parameter } & \multicolumn{1}{c}{ Value } \\
\hline Patch size & $2.1 \times 2.3$ inches \\
\hline Patch separation from ground-plane & 200 mils \\
\hline Parasitic element size - PLXPW & see Table II \\
\hline Parasitic element separation from the patch & $100 \mathrm{mils}$ \\
\hline Patch/parasitic element thickness & $30 \mathrm{mils}$ \\
\hline Feeding network separation from ground-planc & $100 \mathrm{mils}$ \\
\hline 3D Transition width at patch level & $420 \mathrm{mils}$ \\
\hline 3D Transition width at feeding network level & $120 \mathrm{mils}$ \\
\hline 3DTransition length & 1 inch \\
\hline Patch separation H-plane - HS & see Table II \\
\hline Patch separation E-plane - ES & see Table II \\
\hline
\end{tabular}

TABLE II

The Array Performance Summary

\begin{tabular}{|c|c|c|c|c|c|c|c|c|c|c|}
\hline$\overline{\text { Fig. }}$ & $\overline{P L \times P W}$ & HS & ES & $\begin{array}{c}\text { H-plane } \\
\text {-3db Beam } \\
\left.\right|^{\circ}{ }^{\circ}\end{array}$ & $\begin{array}{c}\text { E-plane } \\
\text { 3dB Beam } \\
\left.\right|^{\circ} \mid\end{array}$ & $\begin{array}{l}\text { Hd-plane } \\
\text { Sidelobes } \\
\text { [dB] }\end{array}$ & $\begin{array}{c}\text { E-plane } \\
\text { Sidelobes } \\
{[\text { dB } \mid}\end{array}$ & Front/Back & $\begin{array}{c}\text { VSWR }<2: 1 \\
\text { Bandwidth } \\
{[\mathrm{GHz}]}\end{array}$ & $\begin{array}{c}\text { Gain } \\
@ 2.45 \mathrm{GHz} \\
\text { [dB] }\end{array}$ \\
\hline $8 \mathrm{a}$ & $2.1 \times 4.4$ & 2.75 & 2.75 & 39 & 56 & 25 & 22 & 22 & $2.34-2.54$ & 10.50 \\
\hline $8 \mathrm{~b}$ & $2.1 \times 6.1$ & 4.00 & 4.00 & 35 & 28 & 20 & 18 & 22 & $2.34-2.60$ & 14.50 \\
\hline
\end{tabular}

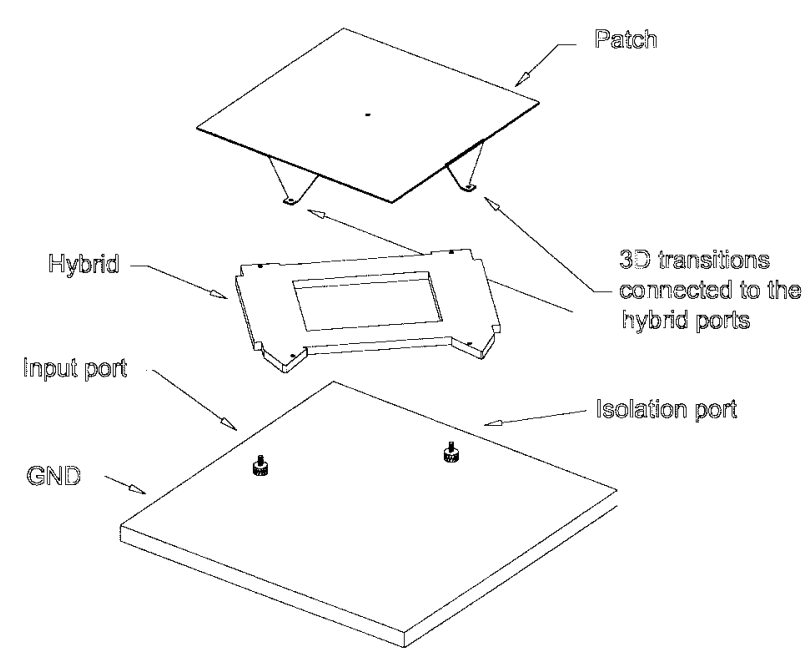

Fig. 6. The circular polarized version of the patch shown in Fig. 3.

These geometries exhibit a number of new design features: First, note that the parasitic elements are connected in $H$ plane. Since the mutual coupling through the common $H$ plane parasitic element was found to be negligible, there is no difference in having two separate parasitic elements or only one covering the two patches; second, the transmission line feeding the lower pair of patches is covered by the common parasitic element. Thus, in large arrays, a significant part of the feeding network can be covered by the parasitic multielements, reducing most of its spurious radiation.

The design parameters for the antennas shown in Fig. 8 are given in Table I and their measured performance is summarized in Table II.

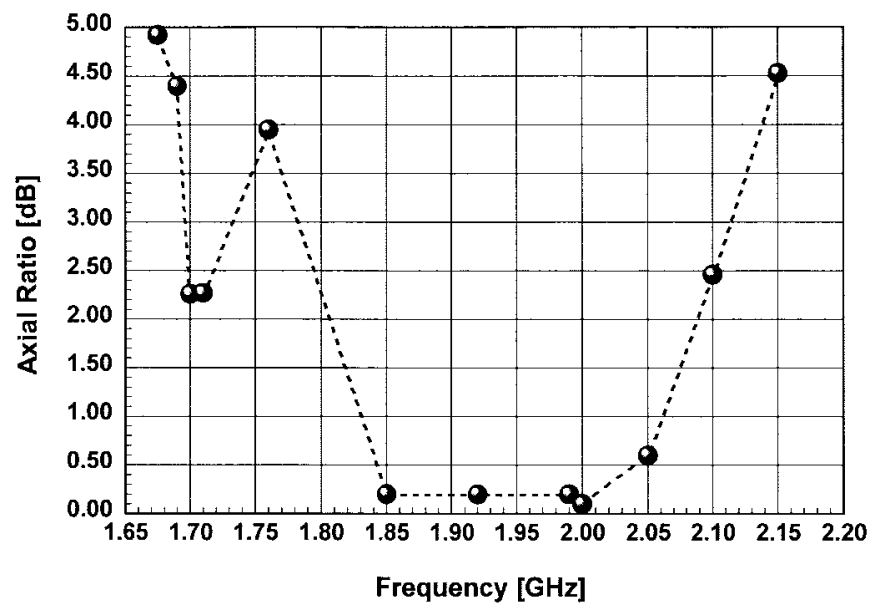

Fig. 7. The axial ratio of the patch shown in Fig. 6.

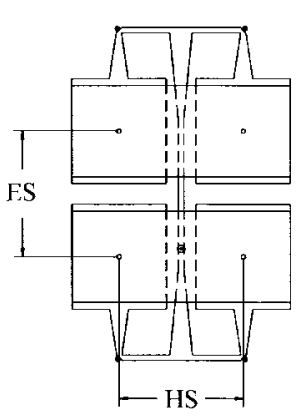

(a)

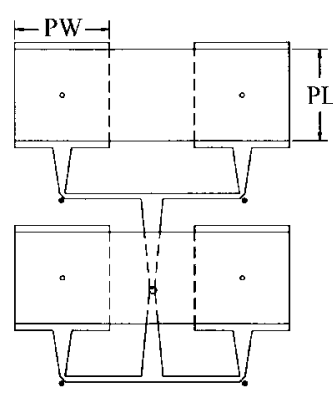

(b)
Fig. 8. Two different designs for a $2 \times 2$ subarray. 
TABLE III

The Serial-Fed Stack-Patch Array Performance Summary. The $H$-Plane Beamwidth is $87^{\circ} \pm 1^{\circ}$ Across the Band

\begin{tabular}{c|c|c|c|c|c}
\hline Frequency & $\begin{array}{c}\text { E-plane } \\
\text { Beamwidth } \\
\mid \mathbf{G H z}]\end{array}$ & $\begin{array}{c}\text { E-plane } \\
\text { Sidelobes } \\
|\mathbf{}|\end{array}$ & Front/Back & Gain & $\begin{array}{c}\text { Squint/ } \\
\text { Beamwidth } \\
\text { Ratio }\end{array}$ \\
\hline 2.40 & 9.5 & 13.2 & 21 & 14 & -0.07 \\
\hline 2.45 & 10.0 & 13.2 & 20. & 15 & 0.00 \\
\hline 2.50 & 10.0 & 12.4 & 21 & 15 & +0.15 \\
\hline
\end{tabular}

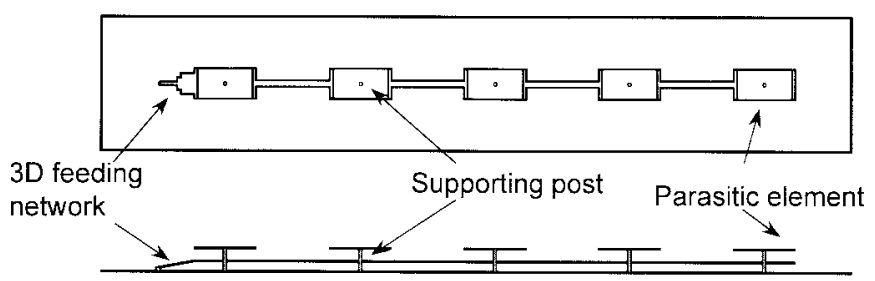

Fig. 9. The serial-fed stacked patch array.

\section{THE Stacked-PATCH ARRAY}

Another application of this technology is the serial-fed stacked-patch array (Fig. 9). This array consists of five serialfed elements and was developed for wireless LAN applications (ISM band). As shown in Table III, the squint across the band is significantly smaller than that of typical serial-fed arrays printed on a dielectric substrate due to the fact that the element phase sensitivity is minimized versus frequency. Since each element supports its own parasitic element, this approach allows an additional degree of freedom in the design. Namely, a certain amplitude/phase distribution can be achieved by loading each driven element with a parasitic element of differing height above the ground plane.

\section{Medium-Gain Dual-Band ARray}

As mentioned above, the main motivation for using this technology is the achievement of high-gain, highly efficient, and inexpensive microstrip arrays. As a first step in this process, a 24-dBi array was designed to work in the high ISM $5.78-\mathrm{GHz}$ band. The layout of this array is shown in Fig. 10. The feeding network is a combination of serial and parallel circuits designed to obtain a very simple layout that mechanically can be supported only by the posts supporting the radiating elements. The design is such that it can incorporate an additional array that was designed to operate in the low ISM 2.4-GHz band. The layout of the two interlaced circuits is shown in Fig. 11. The $S$-band radiating element is a rectangular stacked patch with a high-aspect ratio. The input impedance of this element is $200 \Omega$ so a 3-D linear transformer was required to reduce the impedance to $100 \Omega$. Note that the transformer does not change in width but in height above ground plane. The length of the transformer was determined to match the bandwidth requirements. The radiating elements in this interlaced array introduce a new type of mutual coupling characteristic to this type of nonplanar structure: the $C$-band element is located at 90 mil above the ground plane, while the

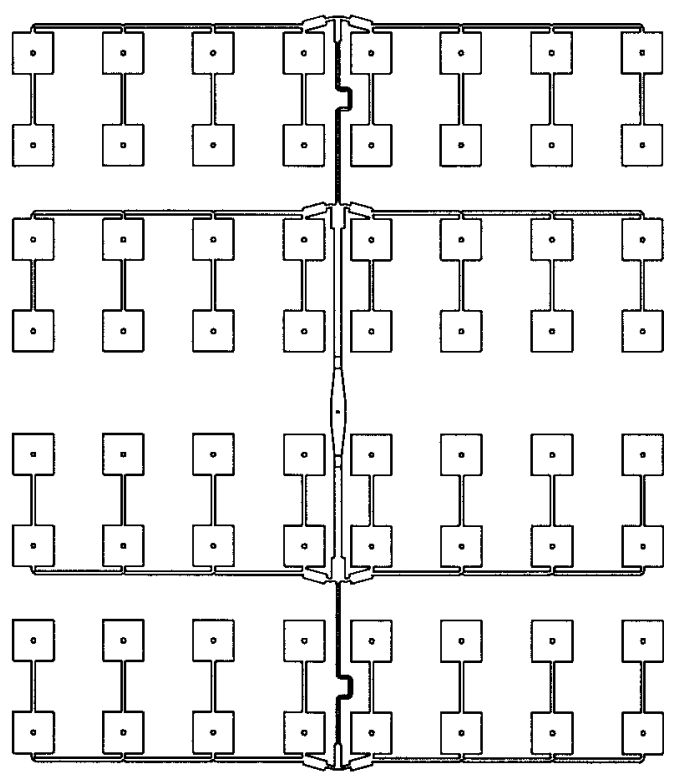

Fig. 10. The layout of the 5.78-GHz medium-gain array.

$S$-band element has a lower patch located at 180 mil above the ground-plane and a parasitic element at $360 \mathrm{mil}$. The mutual coupling between these types of elements was studied using the IE3D simulation package by Zeland software. The results for the specific geometry used in this design showed a level of mutual coupling of about $15 \mathrm{~dB}$ at $S$ band and better than 20 $\mathrm{dB}$ at $C$ band. The presence of the adjacent elements affected the radiation patterns of the individual elements at angles close to grazing, but the effect on the overall array pattern was found to be negligible.

\section{The Patch Antenna with the Bow-Tie Slot}

One way to lower the resonance frequency of a patch is to open an aperture on it. However, as the size of the aperture increases, the bandwidth of the element decreases significantly. By using the concept described in this paper, the patch can be very high (up to a few tenths of a wavelength) off the ground plane to compensate for this reduction in bandwidth. Fig. 12 shows a patch with a bow-tie-shaped slot. The patch is suspended 0.63 in above the ground plane $\left(0.1 \lambda_{\circ}\right)$ and has a VSWR of better than $2: 1$ over the PCS band $(1.85-1.99 \mathrm{GHz}$ $-7.5 \%)$. As shown in Fig. 12, the feeding mechanism is a 3-D suspended microstrip network. Fig. 13 shows the VSWR of the antenna and Fig. 14 shows its radiating patterns. The comparison between Figs. 4 and 13 show similar frequency 


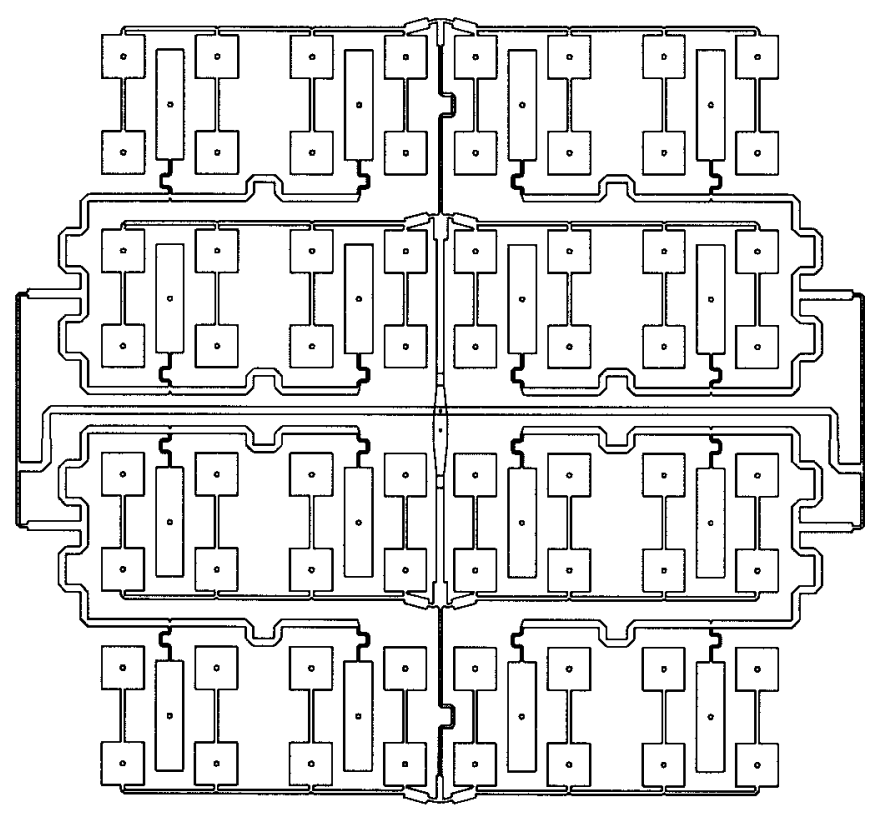

Fig. 11. The layout of the dual-band $(2.45-5.78 \mathrm{GHz})$ array.

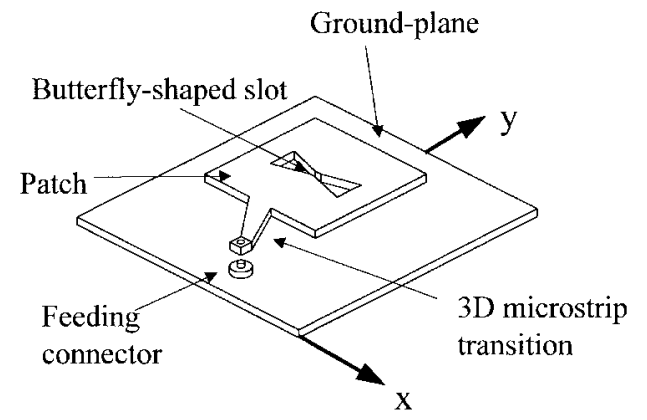

Fig. 12. The patch with the bow-tie slot fed by a 3-D suspended microstrip matching network.

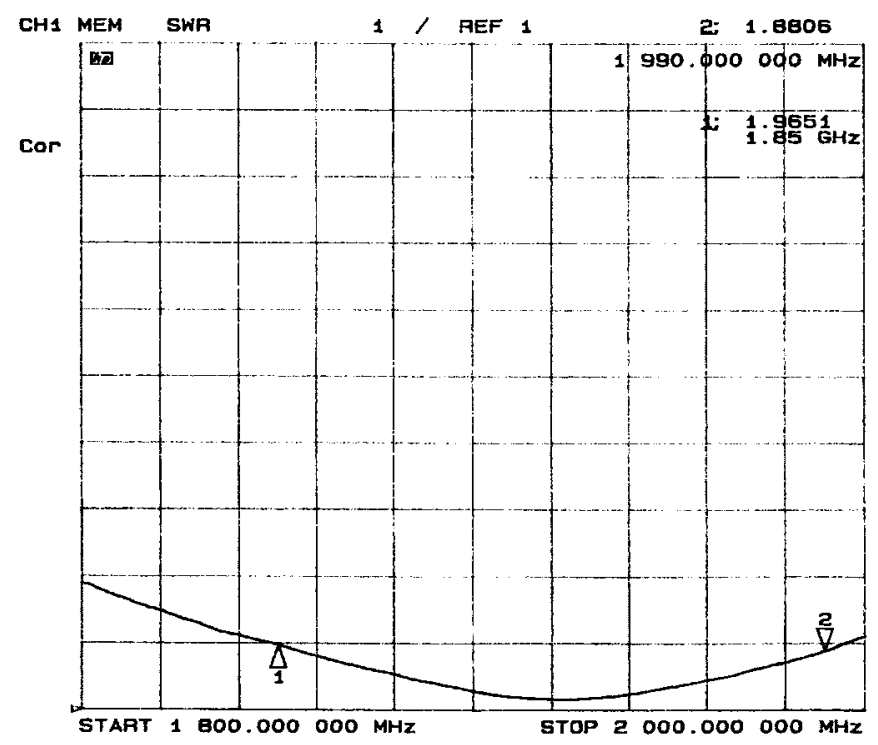

Fig. 13. The VSWR of the antenna shown in Fig. 12.

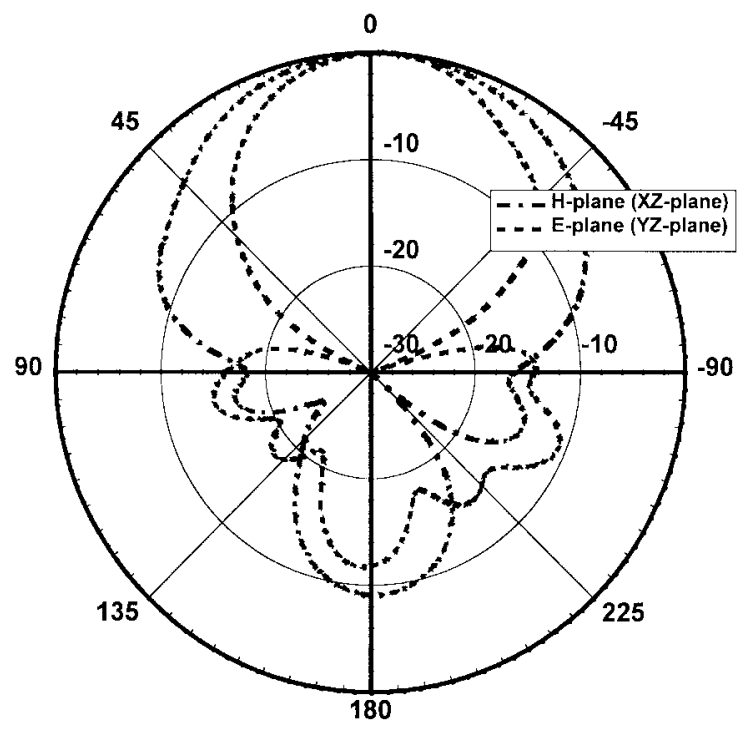

Fig. 14. The radiating patterns of the antennas shown in Fig. 12.

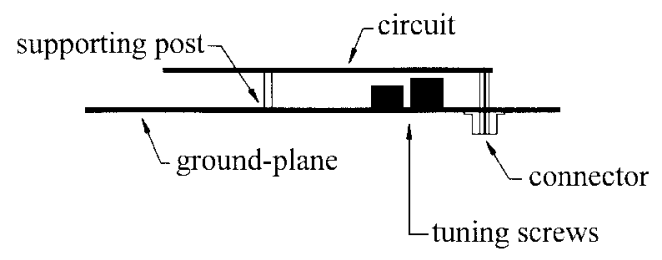

Fig. 15. Waveguide-matching techniques used in microstrip.

responses for the two patches of Figs. 3 and 13, however, the latter patch is about $10 \%$ smaller in size.

\section{MATCHING TEChNiQUES AND MECHANICAL CONSIDERATIONS}

In some applications where the mechanical tolerances cannot be very tight (for cost consideration) final tuning can be done using methods known from waveguide technology. Fig. 15 show a schematic of a sliding-screw tuner similar to those used in waveguides [5], which was used to tune a 24-dB array off in frequency by $0.8 \%$.

Since there is no dielectric substrate or foam for support, the mechanical rigidity of the circuit is achieved through supporting posts that connect the center of the patch to the ground plane. The supporting posts should be nonmetallic for the following reasons: 1) it would be difficult to achieve good electrical contact between the post, the patch, and the ground plane (especially for a large number of elements) and 2) it allows the use of different metals for the circuit and the ground plane while avoiding the bi-metal effect which otherwise would affect the intermodulation performance of the antenna.

An important design parameter that has to be considered here is the metal thickness. In the microstrip antennas printed on dielectric substrates, the metal thickness is small enough to be neglected in the calculation of the characteristic impedance. In our case, since mechanical rigidity is an issue, the thickness of the metal can vary between 15-30 mils. In this case, a 100$\Omega$ line, 90-mil above-ground plane is 143 mil wide if the metal thickness is "zero," 126 mil wide for a 20-mil-thick metal (a 
$13 \%$ difference), and 112 mil wide for a 30-mil-thick metal (a $28 \%$ difference).

\section{CONCLUSIONS}

The suspended microstrip line described in this paper, which allows 3-D transitions, presents new opportunities for the design of flat arrays. The possibility of high-gain arrays with efficiencies comparable to those of the reflector antennas becomes real for frequencies up to possibly $K u$-band. Compared to other types of flat arrays such as waveguide slotted arrays or dipole arrays, the suspended microstrip arrays allow for more bandwidth while considerably reducing the fabrication cost. A variety of designs were presented in this paper that illustrate the freedom of design introduced by this new approach.

\section{ACKNOWLEDGMENT}

The author would like to thank Zeeland Software, Inc., Fremont, CA, for permitting the use of the IE3D simulation package, which served as a design tool for most of the antennas presented in this paper.

\section{REFERENCES}

[1] E. J. Denlinger, "Losses in of microstrip lines," IEEE Trans. Microwave Theory Tech., vol. MTT-28, pp. 513-522, June 1980.

[2] R. A. Pucel, J. D. Masse, and C. P. Hartwig, "Losses in microstrip," IEEE Trans. Microwave Theory Tech., vol. MTT-16, pp. 342-350, June 1968.

[3] E. Levine, G. Malamud, and S. Shrikman, "A study of microstrip array antennas with the feed network," IEEE Trans. Antennas Propagat., vol. 37, pp. 426-434, Apr. 1989.

[4] D. H. Schaubert, private communication.

[5] R. E. Collin, Foundations for Microwave Engineering. New York: McGraw-Hill, 1966, ch. 5

[6] N. Herscovici, "A study of the effect of apertures on patches," to be published.

Naftali Herscovici (S'80-M'92-SM'94), for photograph and biography, see p. 474 of the April 1998 issue of this TRANSACtIONS. 